\title{
MSL-RAD RADIATION ENVIRONMENT MEASUREMENTS
}

Jingnan Guo ${ }^{1, *}$, Cary Zeitlin², Robert F. Wimmer-Schweingruber ${ }^{1}$, Donald M. Hassler ${ }^{3}$, Bent Ehresmann ${ }^{3}$, Jan Köhler ${ }^{1}$, Eckart Böhm ${ }^{1}$, Stephan Böttcher ${ }^{1}$, David Brinza ${ }^{4}$, Sönke Burmeister ${ }^{1}$, Francis Cucinotta ${ }^{5}$, Cesar Martin ${ }^{1}$, Arik Posner ${ }^{6}$, Scot Rafkin ${ }^{3}$ and Guenther Reitz ${ }^{7}$

${ }^{1}$ Christian Albrechts University, Kiel, Germany

${ }^{2}$ Southwest Research Institute, Durham, NH, USA

${ }^{3}$ Southwest Research Institute, Boulder, CO, USA

${ }^{4}$ Jet Propulsion Laboratory, California Institute of Technology, Pasadena, CA, USA

${ }^{5}$ UNLV, Nevada, USA

${ }^{6}$ NASA Headquarters, Washington, DC, USA

${ }^{7}$ DLR, Cologne, Germany

*Corresponding author: guo@physik.uni-kiel.de

\begin{abstract}
In this study, results are presented from the on-board radiation assessment detector (RAD) of Mars Science Laboratory (MSL). RAD is designed to measure the energetic particle radiation environment, which consists of galactic cosmic rays (GCRs) and solar energetic particles (SEPs) as well as secondary particles created by nuclear interactions of primary particles in the shielding (during cruise) or Martian soil and atmosphere (surface measurements). During the cruise, RAD collected data on space radiation from inside the craft, thus allowing for a reasonable estimation of what a human crew travelling to/from Mars might be exposed to. On the surface of Mars, RAD is shielded by the atmosphere (from above) and the planet itself (from below). RAD measures the first detailed radiation data from the surface of another planet, and they are highly relevant for planning future crewed missions. The results for radiation dose and dose equivalent (a quantity most directly related to human health risk) are presented during the cruise phase, as well as on the Martian surface. Dose and dose equivalent are dominated by the continuous GCR radiation, but several SEP events were also detected and are discussed here.
\end{abstract}

\section{INTRODUCTION OF RAD}

The Mars Science Laboratory (MSL) mission was launched on 26 November 2011 and landed on Mars after a 253-d cruise on 6 August 2012. The radiation assessment detector (RAD) on board the Curiosity rover was designed to measure a broad spectrum of energetic particle radiation ${ }^{(1)}$. It combines chargedand neutral-particle detection capabilities over a wide dynamic range in a compact, low-mass, low-power instrument. A schematic view of RAD can be seen in Figure 1. For charged-particle detection, silicon detectors $\mathrm{A}$ and $\mathrm{B}$ are used in coincidence to define the acceptance angle of incoming particles. Highly energetic particles may enter the view cone and penetrate the entire stack before they leave the detector at the bottom. Information on energy deposited in each detector can be recorded, while the total energy of penetrating particles cannot be simply determined. Less-energetic particles may stop in one of the detectors, and their energy, charge and mass can be determined. For neutral-particle detection, both $\mathrm{D}$ and $\mathrm{E}$ detectors are, to different degrees, sensitive to $\gamma$-rays and neutrons. The separation and reconstruction of $\gamma$-ray and neutron energy spectra have been applied to RAD measurements of the Martian surface neutralparticle fluxes ${ }^{(3)}$.

RAD has collected radiation measurements during its cruise to $\mathrm{Mars}^{(4)}$ and now on the surface of
Mars ${ }^{(5)}$. The assessment of the radiation environment is fundamental for planning future human missions to Mars and evaluating the impact of radiation on the preservation of organic bio-signatures.

\section{RADIATION MEASUREMENT DURING CRUISE}

On its way to Mars, RAD obtained 7 months of firsttime measurements of the radiation environment inside the shielding of a spacecraft, in principal similar to those planned for future human exploration to the planet, thus providing insight into the potential radiation hazard for astronauts ${ }^{(4)}$. The total accumulated cruise dose equivalent was about $466 \pm 84 \mathrm{mSv}$ dominated mainly by galactic cosmic rays (GCR), with roughly $5 \%$ caused by five observed solar energetic particle (SEP) events. The results indicate a radiation dose equivalent of $0.66 \pm 0.12$ Sievert employing current propulsion systems and comparable shielding assuming a faster 180 -d one-way duration. Note that these results are a strong function of both the solar output and the shielding surrounding RAD during cruise. Actual astronaut exposures will depend on the habitat shielding, solar and heliospheric modulation, and the unpredictable nature of large solar particle events (SPEs). The SPE contribution 


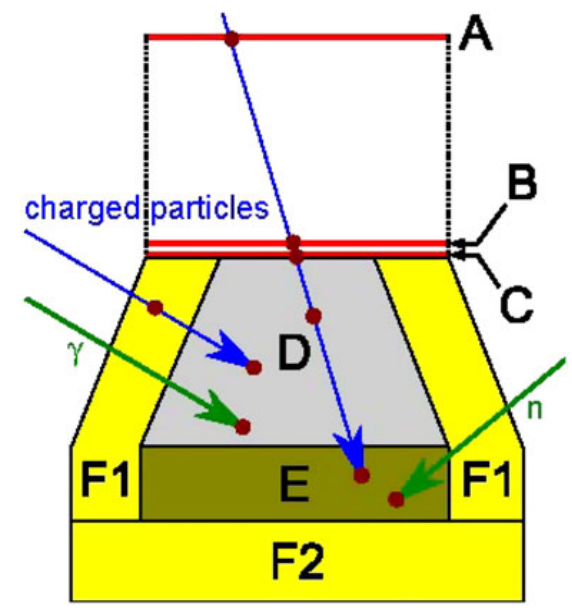

Figure 1. Schematic view of the RAD instrument, consisting of three silicon detectors (A, B and C, each having the thickness of $300 \mathrm{~mm}$ ), a caesium iodide scintillator (D with a height of $28 \mathrm{~mm}$ ) and a plastic scintillator (E with a height of $18 \mathrm{~mm})$. Both scintillators are surrounded by a plastic anticoincidence $(\mathrm{F})$. For detecting charged particles, A, B, C, D and E are used as a telescope. Neutral particles (both neutrons and gammas, green) are detected in $\mathrm{D}$ and $\mathrm{E}$ using $\mathrm{C}$ and $\mathrm{F}$ as anticoincidence. This figure is adapted originally from Figure 3 of Ref. (2).

could conceivably be many times larger in a different time frame.

\section{Dose rate measurements}

RAD dose measurements were taken using two concurrent methods: one with silicon detector $\mathrm{B}$ and the other one with plastic scintillator $\mathrm{E}^{(4)}$. The latter has a composition similar to that of human tissue, and it is also more sensitive to neutrons than silicon detectors. Figure 2 shows the dose rate measurements taken from 6 December 2011 to 14 July 2012 obtained during the cruise. During quiet periods in solar activity, the GCR dose rate measured by the plastic scintillator (red) was about $461 \pm 92 \mu \mathrm{Gy} \mathrm{d}^{-1}$.

The measurement from the silicon detector (black) averaged $332 \pm 23 \mu \mathrm{Gy} \mathrm{d}^{-1}$ after subtraction of the background radiation from Curiosity's radioisotope thermoelectric generator $\left(\approx 85 \mu \mathrm{Gy} \mathrm{d}^{-1}\right)$. To compare with the measured dose rate in plastic and with model predictions, the dose rate in silicon can be converted, approximately, to dose rate in water. A constant factor of 1.45 was applied to relate energy loss per unit of path length $(\mathrm{d} E / \mathrm{d} x)$ in silicon to linear energy transfer (LET) in water ${ }^{(4)}$. After conversion, the dose rate in water, as measured in the silicon detector, was $481 \pm 80 \mu \mathrm{Gy} \mathrm{d}^{-1}$, which is a comparable value within uncertainties to measurements in the plastic scintillator.

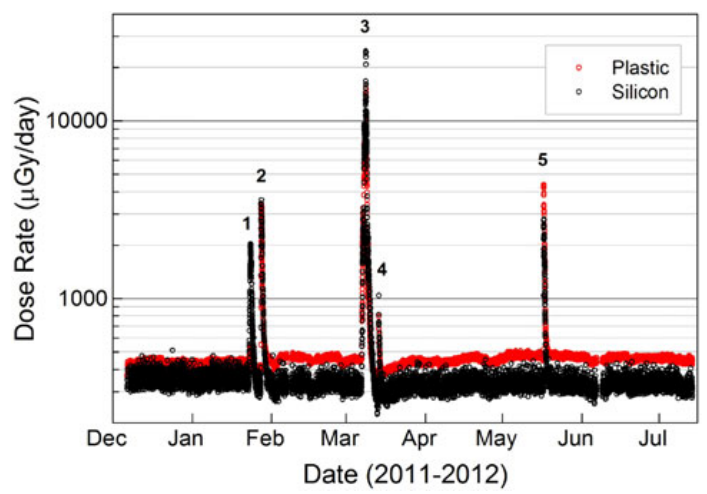

Figure 2. Dose rates recorded in a silicon detector and in a plastic scintillator (tissue equivalent) during the MSL's cruise to Mars. There are five observed SEP events marked with numerals. This figure is adapted from Ref. (4).

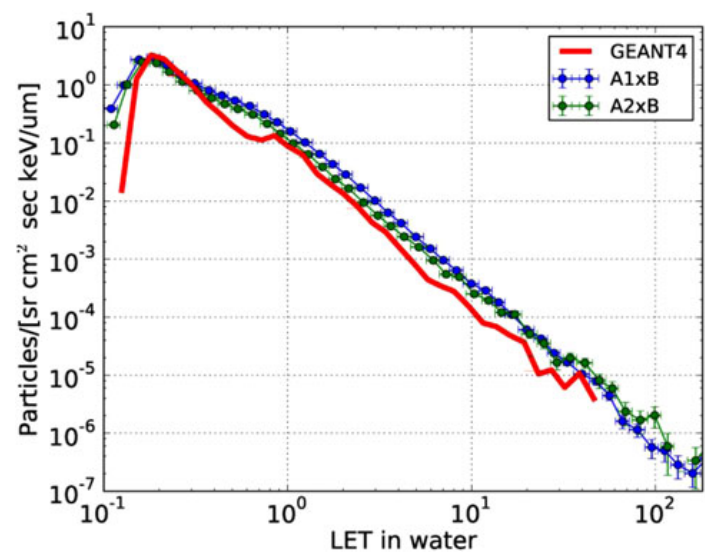

Figure 3. The LET $(\mathrm{d} E / \mathrm{d} x)$ spectrum in water measured using charged-particle coincidence events and obtained from GEANT4 Monte Carlo simulations. Based on two different geometric coincidence conditions (1), there are two onboard LET spectra that mostly agree with each other when converted to flux per geometric angle given an isotropic radiation environment.

\section{LET histogram}

The conventional method of risk estimation uses a quality factor that is a function only of LET in water, $Q($ LET $)$. One of the RAD silicon detectors, detector $\mathrm{B}$, is used to produce an LET spectrum [for a charged particle that satisfies a coincidence path in a telescope configuration, its path length $(\mathrm{d} x)$ can be estimated and its deposited energy $(\mathrm{d} E)$ in the silicon detector is recorded; this generates a $\mathrm{d} E / \mathrm{d} x$ spectrum that can be converted to LET spectrum in water] as shown in Figure 3 using events with coincident hits in A and B. To verify the accuracy of the on-board LET 
histogram, proton and alpha spectra of the BadhwarO'Neill GCR model ${ }^{(6)}$ were used and Monte Carlo simulations were carried out (a GEANT4 ${ }^{(7)}$ Monte Carlo simulation has been set up with RAD geometric configurations and instrumental responses in order to model the process of the particle energy deposit throughout the detector) to forward model the deposited energy spectra in the silicon detector. Figure 3 shows the LET spectrum $(\mathrm{d} E / \mathrm{d} x$ in silicon has been converted to LET in water) measured directly by RAD together with that generated from Monte Carlo simulations. The spectra obtained using forward-modelling methods are comparable with the observations. The discrepancy between the Monte Carlo solutions and the direct observations is due to the lack of noise evaluations and contributions of electrons and heavy ions in the calculations. Given the function of $Q$ (LET), the integrated dose and the dose equivalent can be obtained, which are then used to evaluate the average quality factor ${ }^{(4)}$.

\section{Quality factor and dose equivalent}

From the LET spectrum, an average value of the cruise quality factor was estimated at about $3.82 \pm$ $0.25^{(4)}$. Multiplying the measured average tissue dose rate $\left(\approx 481 \mu \mathrm{Gy} \mathrm{d}^{-1}\right)$ with the average quality factor yields a GCR dose equivalent rate of $1.84 \pm 0.33$ $\mathrm{mSv} \mathrm{d}^{-1}$. In the case of quiet solar conditions, i.e. only considering the contributions from GCR particles, a total dose equivalent during the cruise $(253 \mathrm{~d}$ ) of about $466 \pm 84 \mathrm{mSv}$ have been obtained. If a fast human transit of $360 \mathrm{~d}$ return-trip to Mars is considered, a total dose equivalent of about $662 \mathrm{mSv}$ from GCR particles would be expected during the transit phases of the mission. It is important to note that the measurements depended strongly on heliospheric modulation conditions during the cruise (i.e. near the current solar maximum with low solar activity) and on the spacecraft shielding.

\section{SEP events}

As shown in Figure 2, there are five SEP events observed during the cruise phase. SEP doses were obtained by subtracting average GCR dose rates from the total dose for the duration of the events. Since the SEP fluxes are dominantly protons, for which $Q$ is $\sim 1$, dose and dose equivalent are therefore approximately equal (note that the few secondary neutrons produced by spallation of the shielding material have been disregarded). The integrated equivalent for the five events is about $24.7 \mathrm{mSv}$, which roughly equals to $15 \mathrm{~d}$ of GCR dose equivalent during low solar activity.

\section{RADIATION MEASUREMENTS ON MARS}

In interplanetary space, GCRs consist of $98 \%$ atomic nuclei and $2 \%$ electrons, with the nuclei further divided into about $87 \%$ protons, $12 \%$ helium and only $1 \%$ heavier nuclei ${ }^{(8)}$. GCR intensity and composition, however, vary slightly depending on modulation from the solar cycle phase/Coronal Mass Ejection occurrence rate and to a less extent on synodic (26-d) solar rotation phase. The resulting energetic particle radiation on the Martian surface is to a large extent characterised by these primary GCRs, as well as secondary particles (including neutrons) produced by their interactions in the soil and atmosphere. Therefore, the radiation environment at the surface is determined by the characteristics of the primary radiation at the top of the atmosphere, the composition and mass of the atmosphere, and the composition of the surface soil. In the atmosphere, primary GCRs undergo inelastic interactions with the atmospheric nuclei creating secondary nuclei via spallation and fragmentation processes (especially for heavy ions) producing secondary fragments with lower charge and smaller mass. These interactions occur with primary particles as well as secondary particles as they propagate through the atmosphere. Eventually, these particles reach the planetary surface where they may also penetrate the Martian soil. Secondary neutrons can be generated from the interaction of incident particles within the regolith; when backscattered, they are detected as 'albedo' neutrons ${ }^{(9)}$. Both the downward and upward particle fluxes contribute to the radiation dose rate on the surface of Mars. The surface GCRinduced radiation dose rate measured by RAD has an average value of $210 \pm 40 \mu \mathrm{Gy} \mathrm{d}^{-1}$, and the dose equivalent rate is about $0.64 \pm 0.12 \mathrm{mSv} \mathrm{d}^{-1(5)}$.

The Rover Environmental Monitoring Station $(\mathrm{REMS})^{(10)}$, also on board of MSL, has reported daily thermal tides that can cause variations in the atmospheric pressure as large as $10 \%$ between day and night. RAD has shown that the diurnal variations in radiation dose rate are inversely related to the variations in the atmospheric pressure ${ }^{(11)}$.

\section{PARTICLE TYPES AND FLUXES}

RAD measures mainly downward fluxes on the surface of Mars. RAD separates the charged particles into two categories: particles stopping inside one of the detectors, and particles penetrating through the whole instrument and still creating energy depositions at the bottom detector.

\section{Stopping charged particles}

To identify the ion species of stopping charged particles, RAD makes use of a so-called ' $E_{\mathrm{tot}} \cdot(\mathrm{d} E / \mathrm{d} x)$ ' method, where $\mathrm{d} E / \mathrm{d} x$ means the specific energy loss 
of a particle in unit length of matter and $E_{\text {tot }}$ represents the total energy of the particle. According to the Bethe-Bloch equation $^{(12)}$, the energy loss of a charged particle in a detector can be simplified as

$$
E_{\mathrm{tot}} \cdot(\mathrm{d} E / \mathrm{d} x) \propto M \cdot Z^{2},
$$

where $M$ and $Z$ are the atomic mass and charge of the particle, respectively. The preceding equation can be interpreted as following: the product of the particle's total energy $E_{\text {tot }}$ and the energy loss $\mathrm{d} E$ in a detector (e.g. detector B with a thickness of $300 \mu \mathrm{m}$ ) is constant for particles of the same ion species (with the same $M$ and $Z$ ). Different stopping ion species are separated from another by their differences in $M$ and $Z$. Based on this logic, the stopping particle histogram is plotted and shown in Figure 4, where all particles of the same species (e.g. protons) lie on a line parallel to the $x$-axis. The ' $E_{\mathrm{tot}} \cdot(\mathrm{d} E / \mathrm{d} x)$ ' method can therefore allow to select stopping particles with certain species and obtain their fluxes. The stopping particle spectra measured with RAD on the surface of Gale Crater are presented in Ref. (13).

\section{Penetrating charged particles}

For particles with such high energies that they do not lose significant energy even when passing through the

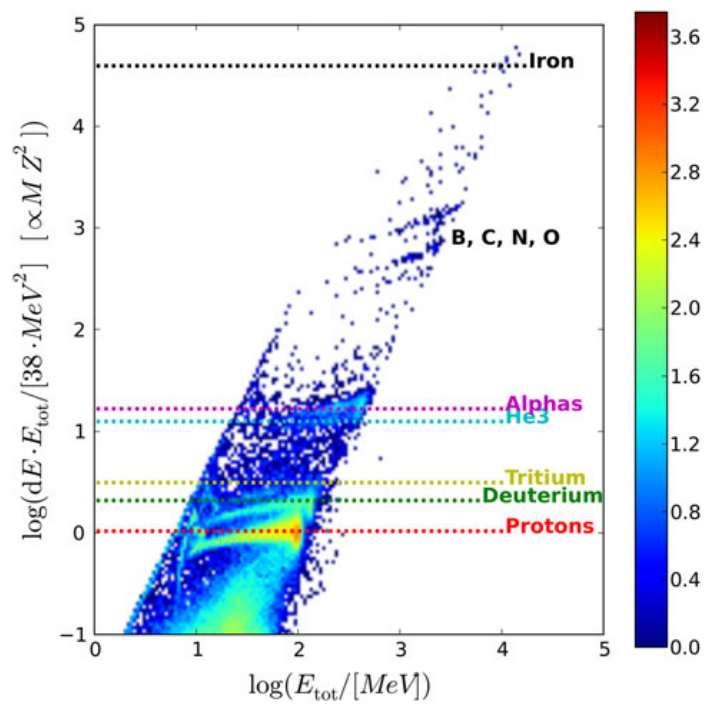

Figure 4. $E_{\mathrm{tot}} \cdot \mathrm{d} E$ versus $E_{\mathrm{tot}}$ histogram in logarithmic scale to separate species of stopping particles, measured by RAD on the surface of Mars during a time period of $250 \mathrm{~d}$. The horizontal dotted lines mark different particles with their $y$ values proportional to $M \cdot Z^{2}$. Note that the $y$-axis was scaled so that $E_{\text {tot }} \cdot \mathrm{d} E$ is one (or zero in logarithmic scale) for protons. The colour bar represents the logarithm of the counts in each bin. instrument, the ratio of energy deposit in the bottom detector $\mathrm{E}$ versus the energy deposit in the front silicon detectors (i.e. detector A, B and C) is nearly constant independent of ion species. For particles that are energetic enough to penetrate the whole instrument but lose a significant amount of energy in detector E, this ratio is slightly higher. According to Equation (1), the energy deposit is proportional to $M \cdot Z^{2} / E_{\text {tot }}$. Although $E_{\text {tot }}$ is unknown for penetrating particles, the general trend is still that energy deposit increases with bigger $M$ and $Z$. Ref. (13) has calibrated the flux of penetrating particles in different species on the surface of Mars based on this method.

\section{Neutral particles}

RAD also has the ability to distinguish between neutrons and $\gamma$-rays due to different detection efficiencies of the two scintillators for these two types of neutral particles. In Ref. (3), an inversion method is used to reconstruct the neutron spectra on the surface of Mars and estimated the resulting neutron dose to be $14 \pm 4 \mu \mathrm{Gy} \mathrm{d}^{-1}$ and a dose equivalent rate of $61 \pm$ $15 \mu \mathrm{Gy} \mathrm{d}^{-1}$. This corresponds to $7 \%$ of the measured total surface dose rate and about $10 \%$ of the biologically relevant surface dose equivalent rate on Mars.

\section{CONCLUSIONS}

RAD measurements have provided the first-ever and representative observations of the radiation environment on the surface of Mars as well as during the cruise to Mars under the current heliospheric conditions (i.e. rising phase and maximum of an unusually weak solar cycle). Data on radiation dose rate and equivalent dose are giving new insight into the potential biological impact on future crewed missions. Furthermore, the detection of charged- and neutralparticle fluxes provides valuable new information for evaluating particle transport processes through the Martian atmosphere.

\section{FUNDING}

RAD is supported by the National Aeronautics and Space Administration (NASA) under JPL subcontract 1273039 to the Southwest Research Institute and by DLR and DLR's Space Administration, grant numbers 50QM0501 and 50QM1201, to the Christian Albrechts University in Kiel, Germany. Part of this research was carried out at JPL under a contact with NASA.

\section{REFERENCES}

1. Hassler, D. M. et al. The radiation assessment detector (RAD) investigation. Space Sci. Rev. 170, 503-512 (2012). 


\section{J. GUO ET AL.}

2. Posner, A., Hassler, D. M., McComas, D. J., Rafkin, S., Wimmer-Schweingruber, R. F., Böhm, E., Böttcher, S., Burmeister, S., Dröge, W. and Heber, B. A high-energy telescope for the Solar Orbiter. Adv. Space Res. 36, 1426-1431 (2005).

3. Köhler, J. et al. Measurements of the neutron spectrum on the Martian surface with MSL/RAD. J. Geophys. Res. Planets 119, 594-603 (2014).

4. Zeitlin, C. et al. Measurements of energetic particle radiation in transit to Mars on the Mars Science Laboratory. Science 340(6136), 1080-1084 (2013).

5. Hassler, D. M. et al. Mars' surface radiation environment measured with the Mars Science laboratory's curiosity rover. Science 343(6169), 1244797 (2014).

6. O'Neill, P. M. Badhwar-O'Neill 2010 galactic cosmic ray flux model — revised. IEEE Trans. Nucl. Sci. 57, 3148 (2010).

7. Agostinelli, S. et al. Geant4-a simulation toolkit. Nucl. Inst. Met. Phys. Res. 506, 250-303 (2003).
8. Simpson, J. A. Elemental and isotopic composition of the galactic cosmic rays. Ann. Rev. Nucl. Sci. 33, 323-382 (1983).

9. Boynton, W. V. et al. The mars odyssey gamma-ray spectrometer instrument suite. In: 2001 Mars Odyssey. Springer Netherlands, pp. 37-83 (2004).

10. Gómez-Elvira, J. et al. REMS: the environmental sensor suite for the Mars Science Laboratory Rover. Space Sci. Rev. 170(1-4), 583-640 (2012).

11. Rafkin, S. C. R. et al. Diurnal variations of radiation dose rate at the surface of mars as observed by the mars science laboratory radiation assessment detector. J. Geophys. Res. Planets 119(6), 1345-1358 (2014).

12. Goulding, F. S. and Harvey, B. G. Identification of nuclear particles. Ann. Rev. Nucl. Sci. 25, 167-240 (1975).

13. Ehresmann, B. et al. Charged particle spectra obtained with the Mars Science Laboratory radiation assessment detector (MSL/RAD) on the surface of Mars. J. Geophys. Res. Planets 119, 468-479 (2014). 